\title{
ON THE ACCURACY OF SURFACE SPLINE APPROXIMATION AND INTERPOLATION TO BUMP FUNCTIONS
}

\author{
AURELIAN BEJANCU JR \\ Department of Applied Mathematics and Theoretical Physics, University of Cambridge, \\ Silver Street, Cambridge CB3 9EW, UK (ab223@damtp.cam.ac.uk)
}

(Received 23 February 2000)

\begin{abstract}
Let $\Omega$ be the closure of a bounded open set in $\mathbb{R}^{d}$, and, for a sufficiently large integer $\kappa$, let $f \in C^{\kappa}(\Omega)$ be a real-valued 'bump' function, i.e. $\operatorname{supp}(f) \subset \operatorname{int}(\Omega)$. First, for each $h>0$, we construct a surface spline function $\sigma_{h}$ whose centres are the vertices of the grid $\mathcal{V}_{h}=\Omega \cap h \mathbb{Z}^{d}$, such that $\sigma_{h}$ approximates $f$ uniformly over $\Omega$ with the maximal asymptotic accuracy rate for $h \rightarrow 0$. Second, if $\ell_{1}, \ell_{2}, \ldots, \ell_{n}$ are the Lagrange functions for surface spline interpolation on the grid $\mathcal{V}_{h}$, we prove that $\max _{x \in \Omega} \sum_{j=1}^{n} \ell_{j}^{2}(x)$ is bounded above independently of the mesh-size $h$. An interesting consequence of these two results for the case of interpolation on $\mathcal{V}_{h}$ to the values of a bump data function $f$ is obtained by means of the Lebesgue inequality.
\end{abstract}

Keywords: multivariable approximation; surface splines; error estimates

AMS 2000 Mathematics subject classification: Primary 41A05; 41A15; 41A25; 41A63

\section{Introduction}

Surface spline interpolation belongs to the class of radial basis function methods for multivariable approximation. Let $d$ be a positive integer and let $\Omega$ be the closure of a bounded open set in $\mathbb{R}^{d}$. In order to describe the surface spline method for interpolation at the vertices of the uniform $d$-dimensional grid $\mathcal{V}_{h}=\Omega \cap h \mathbb{Z}^{d}$ of mesh-size $h>0$, we denote the elements of $\mathcal{V}_{h}$ by $h z_{1}, h z_{2}, \ldots, h z_{n}$, where $\left\{z_{1}, z_{2}, \ldots, z_{n}\right\} \subset \mathbb{Z}^{d}$. Notice that $n=\mathcal{O}\left(h^{-d}\right)$, as $h \rightarrow 0$. For any real parameter $\gamma>0$, define the basis function $\phi:[0, \infty) \rightarrow \mathbb{R}$ by the formula

$$
\phi(r)= \begin{cases}r^{\gamma}, & \text { if } \gamma \notin 2 \mathbb{N}, \\ r^{\gamma} \ln r, & \text { if } \gamma \in 2 \mathbb{N},\end{cases}
$$

and let $m$ be the integer part of $\gamma / 2$. Further, let $\Pi_{m}^{d}$ be the space of polynomials on $\mathbb{R}^{d}$ of total degree not exceeding $m$, let $N=\operatorname{dim} \Pi_{m}^{d}=(d+m) ! /(d ! m !)$, and denote by $\left\{P_{1}, P_{2}, \ldots, P_{N}\right\}$ the monomial basis of $\Pi_{m}^{d}$. We also let $\mathcal{S}_{h}$ be the linear space of functions $s$ of the form

$$
s(x)=\sum_{j=1}^{n} c_{j} \phi\left(\left\|x-h z_{j}\right\|\right)+\sum_{l=1}^{N} c_{n+l} P_{l}(x), \quad x \in \mathbb{R}^{d},
$$


where $\|\cdot\|$ is the Euclidean norm on $\mathbb{R}^{d}$ and the first $n$ real coefficients $c_{1}, c_{2}, \ldots, c_{n+N}$ satisfy the constraints

$$
\sum_{j=1}^{n} c_{j} P_{l}\left(h z_{j}\right)=0, \quad l=1,2, \ldots, N .
$$

Given any arbitrary function $f: \Omega \rightarrow \mathbb{R}$, it is known that, for each sufficiently small $h$, there exists a unique $s_{h} \in \mathcal{S}_{h}$ satisfying

$$
s_{h}\left(h z_{k}\right)=f\left(h z_{k}\right), \quad k=1,2, \ldots, n .
$$

Moreover, this statement remains true if, in the definition of $\mathcal{S}_{h}$, the place of $\Pi_{m}^{d}$ is taken by any other polynomial space $\Pi_{k}^{d}$ with $k \geqslant m$. It has become customary to call $s_{h}$ the surface spline interpolant to the values of $f$ at the vertices of the finite uniform grid $\mathcal{V}_{h}$.

For every parameter $\gamma>0$, the existence and uniqueness of the surface spline interpolant has been established theoretically by Duchon $[6]$ in the general case of scattered interpolation points, using a variational approach. (In the scattered data case, $h$ is replaced by the Hausdorff distance between the set of interpolation points and the domain $\Omega$.) The same result also follows from the work of Micchelli [18], whose arguments apply to any basis function $\phi$ for which the derivative of order $m+1$ of the function $\psi:=\phi(\sqrt{ } \cdot)$ is strictly completely monotonic, i.e. $(-1)^{k} \psi^{(k+m+1)}(r)>0, \forall r>0, \forall k \in \mathbb{N}$. As a consequence of this existence and uniqueness result, for each $j=1,2, \ldots, n$, there exists a unique function $\ell_{j} \in \mathcal{S}_{h}$ satisfying the Lagrange conditions

$$
\ell_{j}\left(h z_{k}\right)=\delta_{k j}, \quad k=1,2, \ldots, n,
$$

where $\delta_{k j}$ is the Kronecker delta. Thus we have the following Lagrange representation formula for the surface spline interpolant $s_{h}$ to the values of $f$ at the vertices of $\mathcal{V}_{h}$ :

$$
s_{h}(x)=\sum_{j=1}^{n} f\left(h z_{j}\right) \ell_{j}(x), \quad x \in \mathbb{R}^{d} .
$$

Note that each $\ell_{j}$ depends on $\Omega, \gamma$ and $h$.

A basic problem from the point of view of approximation theory is to study the accuracy to which $s_{h}$ approximates $f$ over $\Omega$ when $h \rightarrow 0$, under various smoothness assumptions on $f$. This problem and its version for scattered interpolation points have been investigated by Duchon [7], Arcangéli and Rabut [1], Madych and Nelson [14], Wu and Schaback [27], Powell [22], Matveev [16], Light and Wayne [13], Schaback $[\mathbf{2 4}, \mathbf{2 5}]$ and Johnson [9-12], who estimated the dependence on $h$ of the error (or of some of its derivatives) in the uniform or $L^{p}$-norm $(1 \leqslant p<\infty)$ over the domain $\Omega$. Further, Matveev [17] and Bejancu $[\mathbf{2 , 3}]$ proved that the decay of the error as $h \rightarrow 0$ is significantly faster over a compact subset $K$ of the interior of $\Omega$. Specifically, for any sufficiently differentiable function $f$, we have

$$
\max _{x \in K}\left|f(x)-s_{h}(x)\right|=\mathcal{O}\left(h^{\gamma+d}\right), \quad \text { as } h \rightarrow 0,
$$


Table 1. Values of the error $\left|\left(g_{p}-s_{h}\right)\left(\frac{1}{2} h\right)\right|$ for $d=1, \gamma=2$

\begin{tabular}{rcc}
\hline \multicolumn{1}{c}{$h^{-1}$} & $p=4$ & $p=5$ \\
\hline 16 & 0.00052648 & 0.00029267 \\
32 & 0.00021351 & 0.00011937 \\
64 & 0.00007959 & 0.00004445 \\
128 & 0.00002879 & 0.00001607 \\
256 & 0.00001029 & 0.00000574 \\
\hline
\end{tabular}

which matches the maximal convergence rate over $\mathbb{R}^{d}$ obtained by Buhmann [5] and Powell [21, Theorem 8.5] in the ideal case of interpolation on the infinite grid $h \mathbb{Z}^{d}$.

In the present paper, we assume that $f$ is a 'bump' function, i.e. $f$ is non-zero only on a compact subset of the interior of $\Omega$. Under this hypothesis, a natural question is whether the decay rate $h^{\gamma+d}$ of (1.7) can also be attained by the uniform error $\max _{x \in \Omega} \mid f(x)-$ $s_{h}(x) \mid$.

We can check numerically that the answer to this question is negative for $d=1$ and $\gamma=2$ (in which case $\phi(r)=r^{2} \ln r, m=1$ and $N=\operatorname{dim} \Pi_{1}^{1}=2$ ). Indeed, let $\Omega=[0,1]$, $h:=1 /(n-1), n \in\{2,3, \ldots\}$, and, for each positive integer $p$, define the product

$$
g_{p}(x)=10^{p+1}\left[\max \left\{0, x-\frac{1}{4}\right\}\right]^{p}\left[\max \left\{0, \frac{3}{4}-x\right\}\right]^{p}, \quad x \in[0,1] .
$$

Thus $g_{p} \in C^{p-1}(\Omega)$ and $\operatorname{supp}\left(g_{p}\right)=\left[\frac{1}{4}, \frac{3}{4}\right]$. We choose the data function $f:=g_{p}$ and we estimate the magnitude of the uniform error over $\Omega$ by evaluating the error function $e_{h}:=\left|g_{p}-s_{h}\right|$ at $x=\frac{1}{2} h$. The coefficients of the representation of type (1.2) of $s_{h}$ are computed by solving the $(n+2) \times(n+2)$ system given by (1.3) and (1.4). For $p \in\{4,5\}$, Table 1 shows that $e_{h}\left(\frac{1}{2} h\right)$ is reduced by a factor of approximately $2 \sqrt{2}$ when $h^{-1}$ doubles, which corresponds to a decay of magnitude $h^{3 / 2}$. It can also be checked that the same rate of decay is usual for larger values of $p$ or even for $C^{\infty}$ bump functions $f$.

However, the univariate natural spline case $(d=1, \gamma \in 2 \mathbb{N}+1)$ shows that a positive answer to the question above is sometimes possible. Much insight has been obtained recently by Johnson [11] for the multivariable case in which $\gamma$ is a positive integer such that $\gamma+d$ is even, and for the version of the surface spline interpolation method that uses $\Pi_{(\gamma+d) / 2-1}^{d}$ instead of $\Pi_{m}^{d}$ in the definition of $\mathcal{S}_{h}$. His approach is based on the best approximation property that characterizes the surface spline interpolant in the variational framework of Duchon. When applied to the uniform norm of the error and to the grid of interpolation points $\Omega \cap h \mathbb{Z}^{d}$, Johnson's results imply

$$
\max _{x \in \Omega}\left|f(x)-s_{h}(x)\right|=\mathcal{O}\left(h^{\gamma+d / 2}\right), \quad \text { as } h \rightarrow 0,
$$

for a sufficiently differentiable bump function $f$.

In $\S 2$ we prove, under the same restriction on $\gamma$ and $d$, that the maximal rate $\mathcal{O}\left(h^{\gamma+d}\right)$ can hold uniformly over $\Omega$ for bump data functions if interpolation is replaced by approximation with a suitably constructed element of $\mathcal{S}_{h}$ (cf. Theorem 2.1). On the other hand, 
there are no restrictions on $\gamma$ or $d$ in the main result of $\S 3$, which states that the expression $\max _{x \in \Omega} \sum_{j=1}^{n} \ell_{j}^{2}(x)$ is bounded from above independently of the mesh-size $h$ (cf. Theorem 3.1). As a consequence, we obtain a new proof of the convergence rate (1.9) via the Lebesgue inequality (cf. Corollary 3.4). Furthermore, our approach shows that the order of convergence (1.9) may be improved to the maximal one $\mathcal{O}\left(h^{\gamma+d}\right)$, provided that the Lebesgue constant of the surface spline interpolation operator admits an upper bound that is independent of $h$. This conjecture, which is a topic of current research for the author, is based on encouraging numerical evidence (cf. Remark 3.6).

\subsection{Notation}

$\|\cdot\|$ is the Euclidean norm on $\mathbb{R}^{d}, x^{\mathrm{T}} y$ denotes the dot product of two vectors $x$ and $y$ in $\mathbb{R}^{d}, i$ is the usual root of -1 , and $\exp (\cdot)$ is the complex exponential function of base $e$. Also, const. $(\alpha, \beta, \ldots)$ is a generic notation for various constants which depend only on the indicated arguments $\alpha, \beta$, etc.

\section{Approximation of maximal order}

In this section we work under the assumption that $\gamma+d$ is a positive even integer and we relate the error of surface spline interpolation on the finite grid $\Omega \cap h \mathbb{Z}^{d}$ to the error of interpolation on the infinite grid $h \mathbb{Z}^{d}$.

Multivariable interpolation on the cardinal grid $\mathbb{Z}^{d}$ by means of a basis function of the form (1.1) in the case when $\gamma+d$ is even has been considered by Madych and Nelson [15], extending the univariate cardinal spline theory of Schoenberg [26]. In the following, we need a basic result from [15], namely the existence of a unique set $\left\{\mu_{z}: z \in \mathbb{Z}^{d}\right\}$ of real coefficients that satisfy

$$
\left|\mu_{z}\right| \leqslant A \exp (-a\|z\|), \quad \forall z \in \mathbb{Z}^{d},
$$

for some positive constants $A$ and $a$, such that the function $\chi: \mathbb{R}^{d} \rightarrow \mathbb{R}$,

$$
\chi(x)=\sum_{z \in \mathbb{Z}^{d}} \mu_{z} \phi(\|x-z\|), \quad x \in \mathbb{R}^{d},
$$

which is defined by an absolutely and uniformly convergent series on any compact subset of $\mathbb{R}^{d}$, achieves the Lagrange conditions $\chi(0)=1$ and $\chi(z)=0$ for $z \in \mathbb{Z}^{d} \backslash 0$. In addition, the cardinal function $\chi$ has the property $|\chi(x)| \leqslant B \exp (-b\|x\|), \forall x \in \mathbb{R}^{d}$, for some positive constants $B$ and $b$.

A comprehensive treatment of multivariable cardinal interpolation with radial basis functions has been given by Buhmann $[\mathbf{4}, \mathbf{5}]$, whose theory applies to virtually all of the radial basis functions that are in current use. For example, if the parameter $\gamma>0$ in (1.1) is not a positive integer of the parity of $d$, Buhmann established that the corresponding coefficients $\mu_{z}, z \in \mathbb{Z}^{d}$, of the cardinal function $\chi$ decay at least as fast as $\mathcal{O}\left(\|z\|^{-(\gamma+2 d)}\right)$, for large $\|z\|$. He went further to consider interpolation at the vertices of the scaled grid $h \mathbb{Z}^{d}(h>0)$ and, based on polynomial reproduction properties, to derive convergence orders for such a scheme when $h \rightarrow 0$. In the particular case when the parameter $\gamma$ of 
(1.1) is a positive integer of the same parity as $d$, Buhmann's results imply that, for any function $f^{*} \in C^{\gamma+d}\left(\mathbb{R}^{d}\right)$ whose partial derivatives of order $\gamma+d$ are bounded, the sum

$$
I_{h} f^{*}(x):=\sum_{\zeta \in \mathbb{Z}^{d}} f^{*}(h \zeta) \chi\left(h^{-1} x-\zeta\right), \quad x \in \mathbb{R}^{d},
$$

which is absolutely and uniformly convergent in every compact subset of $\mathbb{R}^{d}$, provides not only the interpolation conditions

$$
I_{h} f^{*}(h z)=f^{*}(h z), \quad \forall z \in \mathbb{Z}^{d},
$$

but also the approximation property

$$
\max _{x \in \mathbb{R}^{d}}\left|f^{*}(x)-I_{h} f^{*}(x)\right| \leqslant \text { const. }\left(f^{*}, \gamma\right) h^{\gamma+d}, \quad \text { as } h \rightarrow 0 .
$$

To return to the case of surface spline interpolation on a finite grid, we let $\Omega$ be the closure of a bounded open set in $\mathbb{R}^{d}$ (note that no boundary conditions are imposed on the domain $\Omega$ in this section). For a fixed parameter $\gamma>0$ and for each $h>0$, recall that $\mathcal{S}_{h}$ is the linear space of surface spline functions of the form (1.2)-(1.3) associated with the grid $\Omega \cap h \mathbb{Z}^{d}$.

Theorem 2.1. Assume that $\gamma+d$ is a positive even integer and let $f \in C^{\gamma+d}(\Omega)$ be a bump data function, i.e. $\operatorname{supp}(f)=K$, for some compact set $K \subset \operatorname{int}(\Omega)$. Then, for every sufficiently small $h$, there exists a surface spline approximant $\sigma_{h} \in \mathcal{S}_{h}$, such that

$$
\max _{x \in \Omega}\left|f(x)-\sigma_{h}(x)\right| \leqslant \text { const. }(f, \gamma, \Omega) h^{\gamma+d}, \quad \text { as } h \rightarrow 0 .
$$

Proof. We construct $\sigma_{h}$ by using the above interpolant $I_{h} f^{*}$ to $f^{*}$ on the infinite grid $h \mathbb{Z}^{d}$, where $f^{*} \in C^{\gamma+d}\left(\mathbb{R}^{d}\right)$ is the trivial extension of $f$ to $\mathbb{R}^{d}$ which takes the constant value zero outside $\Omega$. Since $\operatorname{supp}(f)=K$, equation (2.3) becomes

$$
I_{h} f^{*}(x)=\sum_{\zeta \in \mathbb{Z}^{d} \cap h^{-1} K} f(h \zeta) \chi\left(h^{-1} x-\zeta\right), \quad x \in \mathbb{R}^{d} .
$$

Before using (2.2) to substitute the corresponding series for $\chi\left(h^{-1} x-\zeta\right)$ into (2.7), we observe that, for any $z \in \mathbb{Z}^{d}$, we have

$$
\phi\left(\left\|h^{-1} x-z\right\|\right)= \begin{cases}h^{-\gamma} \phi(\|x-h z\|), & \text { if } \gamma \text { is odd, } \\ h^{-\gamma} \phi(\|x-h z\|)-\left(h^{-\gamma} \ln h\right)\|x-h z\|^{\gamma}, & \text { if } \gamma \text { is even. }\end{cases}
$$

Further, when $\gamma$ is even, we use the 'moment' properties of the coefficients $\mu_{z}, z \in \mathbb{Z}^{d}$ (cf. Buhmann [5, p. 245]), namely

$$
\sum_{z \in \mathbb{Z}^{d}} \mu_{z} p(z)=0, \quad p \in \Pi_{d+\gamma-1}^{d}
$$

to deduce

$$
\sum_{z \in \mathbb{Z}^{d}} \mu_{z}\|x-h z\|^{\gamma}=0
$$


Consequently, for any positive integers $\gamma, d$ which have the same parity and for any $\zeta \in \mathbb{Z}^{d},(2.2)$ provides

$$
\begin{aligned}
\chi\left(h^{-1} x-\zeta\right) & =\sum_{z \in \mathbb{Z}^{d}} \mu_{z} \phi\left(\left\|h^{-1} x-\zeta-z\right\|\right) \\
& =\sum_{z \in \mathbb{Z}^{d}} h^{-\gamma} \mu_{z-\zeta} \phi(\|x-h z\|), \quad x \in \mathbb{R}^{d} .
\end{aligned}
$$

Since the index set $\mathbb{Z}^{d} \cap h^{-1} K$ of the sum in (2.7) is finite, we may make the change in the order of summation that provides the formula

$$
\begin{aligned}
I_{h} f^{*}(x) & =\sum_{\zeta \in \mathbb{Z}^{d} \cap h^{-1} K} f(h \zeta) \sum_{z \in \mathbb{Z}^{d}} h^{-\gamma} \mu_{z-\zeta} \phi(\|x-h z\|) \\
& =\sum_{z \in \mathbb{Z}^{d}} \nu_{z} \phi(\|x-h z\|)
\end{aligned}
$$

where

$$
\nu_{z}:=h^{-\gamma} \sum_{\zeta \in \mathbb{Z}^{d} \cap h^{-1} K} f(h \zeta) \mu_{z-\zeta}, \quad z \in \mathbb{Z}^{d} .
$$

The series on the last line of (2.12) is absolutely convergent for any $x \in \mathbb{R}^{d}$, because it is a finite sum of absolutely convergent series. Note that the coefficients $\nu_{z}, z \in \mathbb{Z}^{d}$, depend on $h$. We now write $I_{h} f^{*}(x)$ as

$$
I_{h} f^{*}(x)=I_{h}^{\Omega} f^{*}(x)+\sum_{z \in \mathbb{Z}^{d} \backslash h^{-1} \Omega} \nu_{z} \phi(\|x-h z\|),
$$

where the truncation operator $I_{h}^{\Omega}$ is defined by

$$
I_{h}^{\Omega} f^{*}(x):=\sum_{z \in \mathbb{Z}^{d} \cap h^{-1} \Omega} \nu_{z} \phi(\|x-h z\|), \quad x \in \mathbb{R}^{d},
$$

and we seek to modify $I_{h}^{\Omega} f^{*}$ in order to obtain the required approximant $\sigma_{h}$. First, however, we estimate the difference $I_{h} f^{*}-I_{h}^{\Omega} f^{*}$.

Lemma 2.2. The hypotheses of Theorem 2.1 imply the condition

$$
\max _{x \in \Omega}\left|I_{h} f^{*}(x)-I_{h}^{\Omega} f^{*}(x)\right| \leqslant \text { const. }(f, \gamma, \Omega) h^{\gamma+d}, \quad \text { as } h \rightarrow 0 .
$$

Proof. The essential ingredient is the exponential decay (2.1) of the coefficients $\mu_{z}$, $z \in \mathbb{Z}^{d}$, of the cardinal function $\chi$, which holds only when $\gamma$ and $d$ are positive integers of the same parity. As a consequence of this property, for any positive integer $p$, we have

$$
\left|\mu_{z}\right| \leqslant \text { const. }(d, \gamma, p)\|z\|^{-p}, \quad \forall z \in \mathbb{Z}^{d} \backslash 0 .
$$

We will choose a suitable value of $p$ later in the proof, and until then we shall work with some large enough value of $p$. 
Let $z$ be any element of $\mathbb{Z}^{d} \backslash h^{-1} \Omega$. Using (2.13), (2.17) and the fact that, asymptotically for $h \rightarrow 0$, the set $\mathbb{Z}^{d} \cap h^{-1} K$ has $\mathcal{O}\left(h^{-d}\right)$ elements, we find the estimate

$$
\begin{aligned}
\left|\nu_{z}\right| & \leqslant h^{-\gamma} \sum_{\zeta \in \mathbb{Z}^{d} \cap h^{-1} K}|f(h \zeta)|\left|\mu_{z-\zeta}\right| \\
& \leqslant \text { const. }(d, \gamma, p) h^{-\gamma} \max _{y \in K}|f(y)| \sum_{\zeta \in \mathbb{Z}^{d} \cap h^{-1} K}\|z-\zeta\|^{-p} \\
& \leqslant \text { const. }(f, d, \gamma, p) h^{-d-\gamma} \max _{\zeta \in \mathbb{Z}^{d} \cap h^{-1} K}\|z-\zeta\|^{-p} \\
& \leqslant \text { const. }(f, d, \gamma, p) h^{-d-\gamma} d_{\mathrm{E}}\left(z, h^{-1} K\right)^{-p},
\end{aligned}
$$

where $d_{\mathrm{E}}\left(z, h^{-1} K\right)$ denotes the Euclidean distance from $z$ to the set $h^{-1} K$. Therefore, the sum of expression (2.14) satisfies

$$
\begin{aligned}
\left|\sum_{z \in \mathbb{Z}^{d} \backslash h^{-1} \Omega} \nu_{z} \phi(\|x-h z\|)\right| & \\
& \leqslant \text { const. }(f, d, \gamma, p) \sum_{z \in \mathbb{Z}^{d} \backslash h^{-1} \Omega} h^{-d-\gamma} \frac{|\phi(\|x-h z\|)|}{d_{\mathrm{E}}\left(z, h^{-1} K\right)^{p}} \\
& \leqslant \text { const. }(f, d, \gamma, p) h^{-d-\gamma} \sum_{z \in \mathbb{Z}^{d} \backslash h^{-1} \Omega} \frac{1+|\phi(\|x\|)|+|\phi(\|h z\|)|}{d_{\mathrm{E}}\left(z, h^{-1} K\right)^{p}}
\end{aligned}
$$

where we have used the inequality

$$
\phi(\|x-y\|) \leqslant \text { const. }(d, \gamma)(1+|\phi(\|x\|)|+|\phi(\|y\|)|), \quad \forall x, y \in \mathbb{R}^{d} .
$$

The term $|\phi(\|x\|)|$ that appears in the numerators of (2.19) is bounded above for $x \in \Omega$ by a constant that depends on $\Omega$.

To estimate the term $|\phi(\|h z\|)|$ of (2.19), it is sufficient to assume $h<1$. Consider first the case when $\gamma$ is even. Since $h<1$, we have $h|\ln h|<1$. Further, $\ln h$ and $\ln \|z\|$ have opposite signs and $\ln \|z\|<\|z\|$ for $z \in \mathbb{Z}^{d} \backslash 0$. Therefore

$$
|\phi(\|h z\|)|=h^{\gamma}\|z\|^{\gamma}|\ln h+\ln \|z\|| \leqslant h^{\gamma-1}\|z\|^{\gamma+1}, \quad \forall z \in \mathbb{Z}^{d} .
$$

When $\gamma$ is odd, we also have $|\phi(\|h z\|)|=h^{\gamma}\|z\|^{\gamma} \leqslant h^{\gamma-1}\|z\|^{\gamma+1}$, for all $z \in \mathbb{Z}^{d}$. It follows that, irrespective of the parity of $\gamma$, the term $|\phi(\|h z\|)|$ of each numerator of (2.19) is bounded by

$$
|\phi(\|h z\|)| \leqslant h^{\gamma-1}\|z\|^{\gamma+1} .
$$

Moreover, for any $z \in \mathbb{Z}^{d} \backslash h^{-1} \Omega$, there exists $u_{z} \in h^{-1} K$ such that $d_{\mathrm{E}}\left(z, h^{-1} K\right)=$ $\left\|z-u_{z}\right\|$. Let $\delta=d_{\mathrm{E}}(\partial \Omega, K)>0$ be the Euclidean distance between $K$ and the boundary $\partial \Omega$ of $\Omega$. Since $h^{-1} \delta \leqslant d_{\mathrm{E}}\left(z, h^{-1} K\right)$ for $z \in \mathbb{Z}^{d} \backslash h^{-1} \Omega$, we have

$$
\begin{aligned}
\|z\| & \leqslant\left\|z-u_{z}\right\|+\left\|u_{z}\right\| \\
& \leqslant d_{\mathrm{E}}\left(z, h^{-1} K\right)+\operatorname{const} .(K) h^{-1} \\
& \leqslant\left(1+\text { const. }(K) \delta^{-1}\right) d_{\mathrm{E}}\left(z, h^{-1} K\right), \quad \forall z \in \mathbb{Z}^{d} \backslash h^{-1} \Omega .
\end{aligned}
$$


Using (2.22) and (2.23) to bound the last sum in (2.19), we obtain

$$
\begin{aligned}
& \left|\sum_{z \in \mathbb{Z}^{d} \backslash h^{-1} \Omega} \nu_{z} \phi(\|x-h z\|)\right| \\
& \leqslant \text { const. }(f, \gamma, \Omega, p) h^{-d-\gamma} \sum_{z \in \mathbb{Z}^{d} \backslash h^{-1} \Omega} \frac{1+h^{\gamma-1} d_{\mathrm{E}}\left(z, h^{-1} K\right)^{\gamma+1}}{d_{\mathrm{E}}\left(z, h^{-1} K\right)^{p}} \\
& \leqslant \text { const. }(f, \gamma, \Omega, p) h^{-d-\gamma} \sum_{z \in \mathbb{Z}^{d} \backslash h^{-1} \Omega} \frac{1}{d_{\mathrm{E}}\left(z, h^{-1} K\right)^{p}} \\
& \quad+\text { const. }(f, \gamma, \Omega, p) h^{-d-1} \sum_{z \in \mathbb{Z}^{d} \backslash h^{-1} \Omega} \frac{1}{d_{\mathrm{E}}\left(z, h^{-1} K\right)^{p-\gamma-1}} .
\end{aligned}
$$

Furthermore, since (2.23) and the inequality $h^{-1} \delta \leqslant d_{\mathrm{E}}\left(z, h^{-1} K\right)$ imply $\|z\|+h^{-1} \delta \leqslant$ const. $(\Omega, K) d_{\mathrm{E}}\left(z, h^{-1} K\right)$ for $z \in \mathbb{Z}^{d} \backslash h^{-1} \Omega$, we have

$$
\begin{aligned}
\sum_{z \in \mathbb{Z}^{d} \backslash h^{-1} \Omega} \frac{1}{d_{\mathrm{E}}\left(z, h^{-1} K\right)^{p}} & \leqslant \text { const. }(\Omega, K) \sum_{z \in \mathbb{Z}^{d}} \frac{1}{\left(\|z\|+h^{-1} \delta\right)^{p}} \\
& \leqslant \text { const. }(\Omega, K, p) \int_{\mathbb{R}^{d}} \frac{\mathrm{d} t}{\left(\|t\|+h^{-1} \delta\right)^{p}} \\
& =\operatorname{const.}(\Omega, K, p) \int_{s=h^{-1} \delta}^{\infty} \frac{\mathrm{d} s}{s^{p-d+1}} \\
& =\text { const. }(\Omega, K, p) h^{p-d} .
\end{aligned}
$$

From (2.14), (2.24) and (2.25), we obtain

$$
\begin{aligned}
\max _{x \in \Omega}\left|I_{h} f^{*}(x)-I_{h}^{\Omega} f^{*}(x)\right| & \leqslant \text { const. }(f, \gamma, \Omega, p)\left(h^{p-2 d-\gamma}+c h^{p-2 d-\gamma-2}\right) \\
& \leqslant \text { const. }(f, \gamma, \Omega) h^{d+\gamma},
\end{aligned}
$$

by choosing the value $p=3 d+2 \gamma+2$ in (2.17). The proof of Lemma 2.2 is complete.

We return to the construction of a suitable approximant $\sigma_{h} \in \mathcal{S}_{h}$. We recall that $N$ denotes the dimension of the space $\Pi_{m}^{d},\left\{P_{1}, P_{2}, \ldots, P_{N}\right\}$ is the monomial basis of $\Pi_{m}^{d}$, and $\Omega \cap h \mathbb{Z}^{d}=\left\{h z_{1}, h z_{2}, \ldots, h z_{n}\right\}$, where $n$ depends on $h$. Let $\mathcal{V}=\left\{y_{1}, y_{2}, \ldots, y_{N}\right\}$ be a fixed subset of $\Omega$ such that interpolation on $\mathcal{V}$ from the linear space $\Pi_{m}^{d}$ has a unique solution (for example, $\mathcal{V}$ may be the principal lattice grid of order $m$ in any simplex that is included in $\Omega$, cf. [19]). Then, as in [2, Proof of Proposition 1], there are positive constants $h_{0}, \delta_{0}$ and $\omega_{0}$, which depend only on $\gamma$ and $\Omega$, such that, for every $h \leqslant h_{0}$, there exists a set $\mathcal{J}_{h}$ with $N$ elements, $\mathcal{J}_{h}=\{t(1), t(2), \ldots, t(N)\} \subset\{1,2, \ldots, n\}$, that has the properties $\left\|h z_{t(j)}-y_{j}\right\|<\delta_{0}, j=1,2, \ldots, N$, and

$$
\left|\operatorname{det}\left(P_{k}\left(h z_{t(j)}\right)\right)_{1 \leqslant j, k \leqslant N}\right| \geqslant \omega_{0} .
$$


The last inequality guarantees the existence of a unique solution $\left\{\alpha_{j}: j \in \mathcal{J}_{h}\right\}$ of the system

$$
\sum_{j \in \mathcal{J}_{h}} \alpha_{j} P_{l}\left(h z_{j}\right)=-\sum_{k=1}^{n} \nu_{z_{k}} P_{l}\left(h z_{k}\right), \quad l=1,2, \ldots, N
$$

where the coefficients $\nu_{z}, z \in \mathbb{Z}^{d}$, are defined by formula (2.13). Thus the function

$$
\sigma_{h}(x):=\sum_{k=1}^{n} \nu_{z_{k}} \phi\left(\left\|x-h z_{k}\right\|\right)+\sum_{j \in \mathcal{J}_{h}} \alpha_{j} \phi\left(\left\|x-h z_{j}\right\|\right), \quad x \in \mathbb{R}^{d},
$$

belongs to $\mathcal{S}_{h}$.

On the other hand, the 'moment' conditions (2.9), and the fact that $\mathbb{Z}^{d} \cap h^{-1} K$ is a finite set in (2.13), imply that similar 'moment' conditions are satisfied by the coefficients $\nu_{z}, z \in \mathbb{Z}^{d}$, namely

$$
\sum_{z \in \mathbb{Z}^{d}} \nu_{z} p(z)=0, \quad p \in \Pi_{d+\gamma-1}^{d} .
$$

Thus the right-hand side entries of the system (2.28) can be written as

$$
-\sum_{k=1}^{n} \nu_{z_{k}} P_{l}\left(h z_{k}\right)=\sum_{z \in \mathbb{Z}^{d} \backslash h^{-1} \Omega} \nu_{z} P_{l}(h z), \quad l=1,2, \ldots, N .
$$

Further, for each $l=1,2, \ldots, N$, the method of proof of Lemma 2.2 shows that

$$
\left|\sum_{z \in \mathbb{Z}^{d} \backslash h^{-1} \Omega} \nu_{z} P_{l}(h z)\right| \leqslant \text { const. }(f, \gamma, \Omega) h^{d+\gamma}, \quad \text { as } h \rightarrow 0,
$$

for a sufficiently large choice of $p$ in (2.17). Now the properties (2.27), (2.28), (2.31) and (2.32) imply $\left|\alpha_{j}\right| \leqslant$ const. $(f, \gamma, \Omega) h^{d+\gamma}$, as $h \rightarrow 0$, for all $j \in \mathcal{J}_{h}$. Therefore the definitions (2.15) and (2.29) give

$$
\begin{aligned}
\max _{x \in \Omega}\left|I_{h}^{\Omega} f^{*}(x)-\sigma_{h}(x)\right| & \leqslant \max _{x \in \Omega} \sum_{j \in \mathcal{J}_{h}}\left|\alpha_{j}\right|\left|\phi\left(\left\|x-h z_{j}\right\|\right)\right| \\
& \leqslant \max _{x, y \in \Omega}|\phi(\|x-y\|)| \sum_{j \in \mathcal{J}_{h}}\left|\alpha_{j}\right| \\
& \leqslant \text { const. }(f, \gamma, \Omega) h^{d+\gamma} .
\end{aligned}
$$

Finally, (2.5), (2.16) and (2.33) imply

$$
\begin{aligned}
& \max _{x \in \Omega}\left|f(x)-\sigma_{h}(x)\right|=\max _{x \in \Omega}\left|f^{*}(x)-\sigma_{h}(x)\right| \\
& \leqslant \max _{x \in \Omega}\left|f^{*}(x)-I_{h} f^{*}(x)\right| \\
& \quad+\max _{x \in \Omega}\left|I_{h} f^{*}(x)-I_{h}^{\Omega} f^{*}(x)\right|+\max _{x \in \Omega}\left|I_{h}^{\Omega} f^{*}(x)-\sigma_{h}(x)\right| \\
& \leqslant \operatorname{const.}(f, \gamma, \Omega) h^{d+\gamma}
\end{aligned}
$$

which completes the proof of Theorem 2.1. 
Remark 2.3. The exponent $\gamma+d$ in the approximation order (2.6) is maximal, in the sense that there exists a sufficiently smooth bump data function $f$ for which the left-hand side of (2.6) does not tend to zero faster than $\mathcal{O}\left(h^{\gamma+d}\right)$, as $h \rightarrow 0$ (see Bejancu $[\mathbf{2}, \mathbf{3}]$ ).

Remark 2.4. In the case $d=3$ and $\gamma=1$, but under different hypotheses on the data function, the maximal convergence order $\mathcal{O}\left(h^{4}\right)$ for approximation with the corresponding type of surface splines has also been obtained by Hardy and Nelson [8].

\section{The Lebesgue inequality and kriging functions}

For the purpose of the next theorem, we consider the general case $\gamma>0, d \geqslant 1$. Let $\Omega \subset \mathbb{R}^{d}$ be a bounded closed domain with non-empty interior and, for any sufficiently small $h>0$, recall that $\Omega \cap h \mathbb{Z}^{d}=\left\{h z_{1}, h z_{2}, \ldots, h z_{n}\right\}$, where

$$
n \leqslant \text { const. }(\Omega) h^{-d}, \quad \text { as } h \rightarrow 0 .
$$

Denote by $T_{h}$ the linear operator that associates to each continuous function $f: \Omega \rightarrow \mathbb{R}$ the unique surface spline $T_{h} f:=s_{h} \in \mathcal{S}_{h}$, which satisfies the interpolation conditions (1.4). The induced $\infty$-norm $\left\|T_{h}\right\|_{\infty}$ of this operator has the value

$$
\left\|T_{h}\right\|_{\infty}=\sup \left\{\max _{x \in \Omega}\left|T_{h} f(x)\right|: f \in C(\Omega), \max _{x \in \Omega}|f(x)| \leqslant 1\right\},
$$

and is called the Lebesgue constant of $T_{h}$. Using the Lagrange representation formula (1.6), a standard argument shows that $\left\|T_{h}\right\|_{\infty}$ is finite and that

$$
\left\|T_{h}\right\|_{\infty}=\max _{x \in \Omega} \sum_{j=1}^{n}\left|\ell_{j}(x)\right|,
$$

where $\left\{\ell_{1}, \ell_{2}, \ldots, \ell_{n}\right\} \subset \mathcal{S}_{h}$ is the set of surface spline functions that are defined by the Lagrange conditions (1.5) (recall that each function $\ell_{j}$ depends on $\Omega, \gamma$ and $h$ ). Moreover, since the interpolation operator $T_{h}$ is a linear, bounded and idempotent map with domain $C(\Omega)$ and range $\mathcal{S}_{h}$, we have the Lebesgue inequality (cf. [20, Theorem 3.1])

$$
\max _{x \in \Omega}\left|f(x)-s_{h}(x)\right| \leqslant\left(1+\left\|T_{h}\right\|_{\infty}\right) d_{\infty}\left(f, \mathcal{S}_{h}\right),
$$

where $d_{\infty}\left(f, \mathcal{S}_{h}\right)$ is the least distance from $f$ to an element of $\mathcal{S}_{h}$, in the uniform norm over $\Omega$. The following result will provide an upper estimate on $\left\|T_{h}\right\|_{\infty}$.

Theorem 3.1. Let $\Omega \subset \mathbb{R}^{d}$ be the closure of a connected, open and bounded set, which satisfies a cone property (see Duchon [7] for a suitable definition of the latter condition). Then, for any parameter $\gamma>0$, there exists a constant $h_{0}>0$ such that the surface spline functions $\ell_{1}, \ell_{2}, \ldots, \ell_{n}$, which satisfy the Lagrange equations (1.5) on the grid $\Omega \cap h \mathbb{Z}^{d}$, have the property

$$
\max _{x \in \Omega} \sum_{j=1}^{n} \ell_{j}^{2}(x) \leqslant \text { const. }(\gamma, \Omega), \quad \forall h \leqslant h_{0} .
$$


Proof. We use a well-known property of the so-called kriging function associated with the grid $\Omega \cap h \mathbb{Z}^{d}$. For a fixed parameter $\gamma>0$ and for each sufficiently small $h>0$, the kriging function $\mathcal{P}_{h}: \mathbb{R}^{d} \rightarrow[0, \infty)$ is given by (cf. Wu and Schaback $[\mathbf{2 7}]$ )

$$
\mathcal{P}_{h}^{2}(x):=\int_{\mathbb{R}^{d}}\left|\Theta_{x}(t)\right|^{2}\|t\|^{-\gamma-d} \mathrm{~d} t, \quad x \in \mathbb{R}^{d},
$$

where

$$
\Theta_{x}(t)=\exp \left(\mathrm{i} x^{\mathrm{T}} t\right)-\sum_{j=1}^{n} \ell_{j}(x) \exp \left(\mathrm{i} h z_{j}^{\mathrm{T}} t\right), \quad t \in \mathbb{R}^{d} .
$$

In order to show that the above integral is finite for each $x \in \mathbb{R}^{d}$, we establish the conditions

$$
\left|\Theta_{x}(t)\right|= \begin{cases}\mathcal{O}\left(\|t\|^{m+1}\right), & \text { for }\|t\| \rightarrow 0, \\ \mathcal{O}(1), & \text { for }\|t\| \rightarrow \infty .\end{cases}
$$

Indeed, $\Theta_{x}$ is bounded for $\|t\| \rightarrow \infty$, being a trigonometric polynomial. Further, the uniqueness of the surface spline interpolation method and (1.6) imply that, for any $p \in$ $\Pi_{m}^{d}$, we have

$$
p(x)=\sum_{j=1}^{n} p\left(h z_{j}\right) \ell_{j}(x), \quad x \in \mathbb{R}^{d} .
$$

Thus, the Taylor expansion of the exponential and (3.9) provide the bound (3.8) for $\|t\|$ near zero. Consequently, the function $g:=\left|\Theta_{x}(\cdot)\right|^{2}\|\cdot\|^{-d-\gamma}$, defined a.e. on $\mathbb{R}^{d}$ (everywhere except the origin), satisfies

$$
g(t)= \begin{cases}\mathcal{O}\left(\|t\|^{2 m+2-\gamma-d}\right), & \text { for }\|t\| \rightarrow 0, \\ \mathcal{O}\left(\|t\|^{-d-\gamma}\right), & \text { for }\|t\| \rightarrow \infty .\end{cases}
$$

Since $m+1>\gamma / 2$, we have $g \in L^{1}\left(\mathbb{R}^{d}\right)$, so the integral (3.6) is finite, as required.

Using the change of variables $v=h t$ in (3.6), we find

$$
\mathcal{P}_{h}^{2}(x)=h^{\gamma} \int_{\mathbb{R}^{d}}\left|\Theta_{x}\left(h^{-1} v\right)\right|^{2}\|v\|^{-d-\gamma} \mathrm{d} v .
$$

Also, the cone condition on $\Omega$ implies the existence of $h_{0}>0$ such that the following estimate holds (cf. Wu and Schaback [27] and Light and Wayne [13]):

$$
\max _{x \in \Omega} \mathcal{P}_{h}^{2}(x) \leqslant \text { const. }(\gamma, \Omega) h^{\gamma}, \quad \forall h \leqslant h_{0} .
$$

Note that, if $\gamma \in(0,2)$, then (3.12) can be established without assuming the cone condition for $\Omega$, as demonstrated by the author in $[\mathbf{3}, \S 5.3]$. The last two displays imply

$$
\max _{x \in \Omega} \int_{\mathbb{R}^{d}}\left|\Theta_{x}\left(h^{-1} v\right)\right|^{2}\|v\|^{-d-\gamma} \mathrm{d} v \leqslant \text { const. }(\gamma, \Omega), \quad \forall h \leqslant h_{0} .
$$


Further, since $1 \leqslant(\pi \sqrt{d})\|v\|^{-1}$ holds for any $v \in[-\pi, \pi]^{d}, v \neq 0$, we deduce

$$
\begin{aligned}
\int_{[-\pi, \pi]^{d}}\left|\Theta_{x}\left(h^{-1} v\right)\right|^{2} \mathrm{~d} v & \leqslant(\pi \sqrt{d})^{\gamma+d} \int_{[-\pi, \pi]^{d}}\left|\Theta_{x}\left(h^{-1} v\right)\right|^{2}\|v\|^{-d-\gamma} \mathrm{d} v \\
& \leqslant(\pi \sqrt{d})^{\gamma+d} \int_{\mathbb{R}^{d}}\left|\Theta_{x}\left(h^{-1} v\right)\right|^{2}\|v\|^{-d-\gamma} \mathrm{d} v .
\end{aligned}
$$

From (3.13), (3.14) and the triangle inequality for $L^{2}$-norms, we obtain

$$
\begin{gathered}
\left\{\int_{[-\pi, \pi]^{d}}\left|\sum_{j=1}^{n} \ell_{j}(x) \exp \left(\mathrm{i} z_{j}^{\mathrm{T}} v\right)\right|^{2} \mathrm{~d} v\right\}^{1 / 2} \\
\quad \leqslant\left\{\int_{[-\pi, \pi]^{d}}\left|\Theta_{x}\left(h^{-1} v\right)\right|^{2} \mathrm{~d} v\right\}^{1 / 2}+\left\{\int_{[-\pi, \pi]^{d}}\left|\exp \left(\mathrm{i} h^{-1} x^{\mathrm{T}} v\right)\right|^{2} \mathrm{~d} v\right\}^{1 / 2} \\
\leqslant \operatorname{const.}(\gamma, \Omega)+(2 \pi)^{d / 2}, \quad \forall h \leqslant h_{0} .
\end{gathered}
$$

On the other hand, since $z_{j} \in \mathbb{Z}^{d}, j=1,2, \ldots, n$, the orthogonality of the trigonometric polynomials provides

$$
\begin{aligned}
\int_{[-\pi, \pi]^{d}}\left|\sum_{j=1}^{n} \ell_{j}(x) \exp \left(\mathrm{i} z_{j}^{\mathrm{T}} v\right)\right|^{2} \mathrm{~d} v & =\int_{[-\pi, \pi]^{d}} \sum_{j=1}^{n} \sum_{k=1}^{n} \ell_{j}(x) \ell_{k}(x) \exp \left(\mathrm{i}\left(z_{j}-z_{k}\right)^{\mathrm{T}} v\right) \mathrm{d} v \\
& =\sum_{j=1}^{n} \sum_{k=1}^{n} \ell_{j}(x) \ell_{k}(x) \int_{[-\pi, \pi]^{d}} \exp \left(\mathrm{i}\left(z_{j}-z_{k}\right)^{\mathrm{T}} v\right) \mathrm{d} v \\
& =(2 \pi)^{d} \sum_{j=1}^{n} \ell_{j}^{2}(x) .
\end{aligned}
$$

Therefore (3.15) and (3.16) imply the required conclusion (3.5).

Remark 3.2. Since $\sum_{j=1}^{n} \ell_{j}^{2}\left(h z_{k}\right)=1, \forall k \in\{1,2, \ldots, n\}$, we have the lower bound $1 \leqslant \max _{x \in \Omega} \sum_{j=1}^{n} \ell_{j}^{2}(x)$. It follows that (3.5) captures the true asymptotic behaviour of $\max _{x \in \Omega} \sum_{j=1}^{n} \ell_{j}^{2}(x)$, as $h \rightarrow 0$.

Remark 3.3. Bounds on the expression $\sum_{j=1}^{n} \ell_{j}^{2}(x)$ have also been considered by Schaback [23] for more general sets of interpolation points. For the case of interpolation at the vertices of the grid $\Omega \cap h \mathbb{Z}^{d}$, the upper bounds of [23] can be made independent of $h$ only if the minimum distance from $x$ to any one of the interpolation points is greater than a constant times $h$. The advantage of the bound (3.5) is that it holds uniformly for $x \in \Omega$.

The following error estimate is an application of Theorems 2.1 and 3.1 and the Lebesgue inequality.

Corollary 3.4. Let $\gamma \in \mathbb{N} \backslash 0$ be such that $\gamma+d$ is even and let $\Omega \subset \mathbb{R}^{d}$ be the closure of a connected, open and bounded set, which satisfies a cone condition. Let $f \in C^{\gamma+d}(\Omega)$ 
Table 2. Evaluations of the Lebesgue constant for $d=\gamma=2$

\begin{tabular}{ccccc}
\hline$h^{-1}$ & 4 & 8 & 16 & 32 \\
\hline$\left\|T_{h}\right\|_{\infty}$ & 1.754925 & 1.894548 & 1.904956 & 1.904987 \\
\hline
\end{tabular}

and assume that $\operatorname{supp}(f) \subset \operatorname{int}(\Omega)$. Further, let $s_{h} \in \mathcal{S}_{h}$ be the surface spline that interpolates $f$ on $\Omega \cap h \mathbb{Z}^{d}$. Then

$$
\max _{x \in \Omega}\left|f(x)-s_{h}(x)\right| \leqslant \text { const. }(f, \gamma, \Omega) h^{\gamma+d / 2}, \quad \text { as } h \rightarrow 0 .
$$

Proof. Combining the discrete Cauchy-Schwarz inequality

$$
\left\{\sum_{j=1}^{n}\left|\ell_{j}(x)\right|\right\}^{2} \leqslant n \sum_{j=1}^{n} \ell_{j}^{2}(x)
$$

with (3.1) and (3.5), we obtain

$$
\max _{x \in \Omega} \sum_{j=1}^{n}\left|\ell_{j}(x)\right| \leqslant \text { const. }(\gamma, \Omega) h^{-d / 2}, \quad \forall h \leqslant h_{0} .
$$

Thus the bound (3.17) on the error of interpolation follows from Theorem 2.1 and the Lebesgue inequality (3.4).

Remark 3.5. We note that Theorem 2.1 and Corollary 3.4 (respectively, Theorem 3.1) remain valid if the polynomial space $\Pi_{m}^{d}$ used in the definition of $\mathcal{S}_{h}$ is replaced by $\Pi_{k}^{d}$, for $m \leqslant k \leqslant \gamma+d-1$ (respectively for any $k \geqslant m$ ). As mentioned in $\S 1$, Johnson [11] has recently obtained the convergence rate (3.17) by different means in the scattereddata case, for the variant of the surface spline interpolation scheme that uses $\Pi_{(\gamma+d) / 2-1}^{d}$ instead of $\Pi_{m}^{d}$ in the definition of $\mathcal{S}_{h}$.

Remark 3.6. Our approach based on the Lebesgue inequality shows that the maximal rate $\mathcal{O}\left(h^{\gamma+d}\right)$ may be obtained in (3.17), provided that $\left\|T_{h}\right\|_{\infty}$ is bounded above by a constant independent of $h$. This conjecture is supported by the numerical results of Table 2 , in which we consider thin plate spline interpolation $(d=\gamma=2)$ at the vertices of the grid $\Omega \cap h \mathbb{Z}^{d}$ on the unit square $\Omega=[0,1] \times[0,1]$. We evaluate the Lebesgue constant (3.3) by computing the maximum of $\sum_{j=1}^{n}\left|\ell_{j}(x)\right|$ for $x \in \Omega \cap\left(\frac{1}{2} h\right) \mathbb{Z}^{d}$. The table indicates a tendency of the values of the Lebesgue constant to remain bounded above by a number smaller than 2 .

Acknowledgements. This work was supported by Trinity College and Clare College, Cambridge, and is based on a part of my $\mathrm{PhD}$ dissertation. I thank my supervisor, Professor M. J. D. Powell, for suggestions which inspired the method of proof of Theorem 2.1. 


\section{References}

1. R. ArCAngÉli And C. Rabut, Sur l'erreur d'interpolation par fonctions splines, Math. Modelling Numer. Analysis 20 (1986), 191-201.

2. A. BEJANCU, Local accuracy for radial basis function interpolation on finite uniform grids, J. Approx. Theory 99 (1999), 242-257.

3. A. Bejancu, Convergence properties of surface spline interpolation, $\mathrm{PhD}$ dissertation, University of Cambridge (1999).

4. M. D. Buhmann, Multivariable interpolation using radial basis functions, $\mathrm{PhD}$ dissertation, University of Cambridge (1989).

5. M. D. Buhmann, Multivariate cardinal interpolation with radial-basis functions, Constr. Approx. 6 (1990), 225-255.

6. J. Duchon, Splines minimizing rotation-invariant seminorms in Sobolev spaces, in Constructive theory of functions of several variables (ed. W. Schempp and K. Zeller), Lecture Notes in Mathematics, no. 571, pp. 85-100 (Springer, 1977).

7. J. Duchon, Sur l'erreur d'interpolation des fonctions de plusieurs variables par les $D^{m}$ splines, R.A.I.R.O. An. Num. 12 (1978), 325-334.

8. R. L. HARdy AND S. A. Nelson, Approximation of Newtonian potentials, in Approximation theory (ed. C. K. Chui, L. L. Schumaker and J. D. Ward), vol. V, pp. 375-378 (Academic, 1986).

9. M. J. Johnson, A bound on the approximation order of surface splines, Constr. Approx 14 (1998), 429-438.

10. M. J. Johnson, An improved order of approximation for thin-plate spline interpolation in the unit disc, Numer. Math. 84 (2000), 451-474.

11. M. J. Johnson, On the error in surface spline interpolation of a compactly supported function, preprint, Kuwait University (1998).

12. M. J. Johnson, The $L_{2}$-approximation order of surface spline interpolation, preprint, Kuwait University (1999).

13. W. Light And H. WAYne, On power functions and error estimates for radial basis function interpolation, J. Approx. Theory 92 (1998), 245-266.

14. W. R. MADYCH AND S. A. NELSON, Multivariate interpolation and conditionally positive definite functions, Approx. Theory Appl. 4 (1988), 77-89.

15. W. R. Madych and S. A. Nelson, Polyharmonic cardinal splines, J. Approx. Theory 60 (1990), 141-156.

16. O. V. MAtveEv, Spline interpolation of functions of several variables and bases in Sobolev spaces, Proc. Steklov Inst. Math. 198 (1994), 119-146.

17. O. V. Matveev, On a method for interpolating functions on chaotic nets, Math. Notes 62 (1997), 339-349. (Transl. Mat. Zametki 62 (1997), 404-417.)

18. C. A. MiCCHELLI, Interpolation of scattered data: distance matrices and conditionally positive definite functions, Constr. Approx. 2 (1986), 11-22.

19. R. A. NiCOLAides, On a class of finite elements generated by Lagrange interpolation, SIAM J. Numer. Analysis 9 (1972), 435-445.

20. M. J. D. Powell, Approximation theory and methods (Cambridge University Press, 1981).

21. M. J. D. PowelL, The theory of radial basis function approximation in 1990, in Advances in numerical analysis, vol. II: Wavelets, subdivision algorithms, and radial basis functions (ed. W. A. Light), pp. 105-210 (Clarendon, Oxford, 1992).

22. M. J. D. Powell, The uniform convergence of thin plate spline interpolation in two dimensions, Numer. Math. 68 (1994), 107-128.

23. R. SCHABACK, Error estimates and condition numbers for radial basis function interpolation, Adv. Comp. Math. 3 (1995), 251-264. 
24. R. SchABACK, Approximation by radial basis functions with finitely many centers, Constr. Approx. 12 (1996), 331-340.

25. R. ScHABACK, Improved error bounds for scattered data interpolation by radial basis functions, Math. Comp. 68 (1999), 201-216.

26. I. J. Schoenberg, Contributions to the problem of approximation of equidistant data by analytic functions, Q. Appl. Math. 4 (1946), 45-99.

27. Z.-M. WU AND R. SCHABACK, Local error estimates for radial basis function interpolation of scattered data, IMA J. Numer. Analysis 13 (1993), 13-27. 